\title{
Chemically vapor deposited diamond films as dosimetric material for potential clinical applications
}

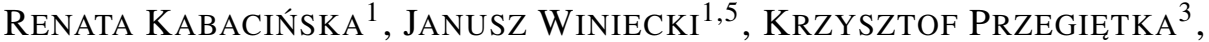 \\ MirosŁaW SZYbowicz ${ }^{4}$, KAZIMIERZ FABisiaK ${ }^{1,2}$, KAZIMIERZ PAProcKI ${ }^{2, *}$ \\ ${ }^{1}$ Oncology Center, Medical Physics Department, Bydgoszcz, Poland \\ ${ }^{2}$ Institute of Physics, Kazimierz Wielki University, Bydgoszcz, Poland \\ ${ }^{3}$ Institute of Physics, Nicolaus Copernicus University, Torun, Poland \\ ${ }^{4}$ Faculty of Technical Physics, Poznan University of Technology, Poznan, Poland \\ ${ }^{5}$ Chair and Clinic Oncology and Radiotherapy, Nicolaus Copernicus University, Torun, Poland
}

\begin{abstract}
Thermally stimulated luminescence (TL), cathodoluminescence (CL) and Raman spectroscopy of CVD diamond films grown on silicon substrates have been studied in order to obtain information on defects created during the growth, which induce the levels within the gap. TL between $300 \mathrm{~K}$ and $700 \mathrm{~K}$, and CL from $200 \mathrm{~nm}$ to $1200 \mathrm{~nm}$ have been measured. The glow curves show a peak located around $610 \mathrm{~K}$ with different intensities, depending on the sample thickness, associated with a trap of energy, equal to $0.83 \mathrm{eV}$ and with attempt-to-escape-time of the order of $10^{8} \mathrm{~s}^{-1}$. Broad CL bands observed at $428 \pm 1 \mathrm{~nm}$ $(2.90 \pm 0.01 \mathrm{eV})$ and $500 \pm 1 \mathrm{~nm}(2.47 \pm 0.004 \mathrm{eV})$ are attributed to closely spaced and widely separated donor-acceptor (D-A) pairs, respectively. The TL and CL results were correlated with diamond quality estimated from Raman spectroscopy measurements.
\end{abstract}

Keywords: CVD diamond; thermoluminescence; Raman spectroscopy; cathodoluminescence

\section{Introduction}

Dosimetry and radiation detection are important, among the other, in hospital X-ray imaging and radiotherapy. Dosimetry is commonly used during system calibration to assess features such as dose or dose rate, dose depth curves, and beam profiles [1]. The dosimetric data obtained during beam calibration are then used during treatment planning, which allows one to ensure optimal delivery of radiation exactly to the place of cancer and protecting in the same time the healthy tissue.

For radiotherapy, an ideal dosimeter should have the following features: high accuracy, high precision, low detection limit, ability to detect radiation over an appropriate dose range, linear dose response which should be independent of the dose rate, independent of the radiation energy, should

\footnotetext{
*E-mail: paprocki@ukw.edu.pl
}

allow the measurement of doses in a very small volume (high spatial resolution) [1, 2]. Diamond seems to be ideal candidate for applications in radiotherapy because its tissue equivalence; the atomic number of carbon $(Z=6)$ is close to that of tissue $(Z=7.4)$. Due to the diamond's high atomic density it should be possible to construct small volume detectors with high spatial resolution.

Recently, some studies underlined convenient TL properties of CVD diamond for radiotherapy $[3,4]$ and highlighted many advantages compared to the commercially available thermoluminescent dosimeters (TLD) generally used in radiotherapy centers [5]. The aim of this work is characterization of diamond films using Raman spectroscopy, TL and CL in terms of their potential use as dosimetric materials. TL and CL are excellent methods for testing homogeneity of CVD layer in terms of defects, impurity content, morphology, and microstructure. 


\section{Materials and methods}

\subsection{Samples preparation}

Diamond films are polycrystalline in nature with a thicknesses of about $2.5 \mu \mathrm{m}$ to $3.5 \mu \mathrm{m}$. They were deposited on a ( $\left.\begin{array}{lll}1 & 0 & 0\end{array}\right)$ silicon substrate by the hot filament chemical vapor deposition (HF CVD) technique. A mixture of methanol and hydrogen $\left(\mathrm{CH}_{3} \mathrm{OH} / \mathrm{H}_{2}=1 \%\right)$ was used as a working gas. The parameters of the growth process were as follows: the total pressure in the reaction chamber $\mathrm{p}=8 \times 10^{3} \mathrm{~Pa}$, substrate temperature: $1100 \mathrm{~K}$ and the working gas flow rate: $100 \mathrm{sccm}$ (standard cubic centimeter per minute). More details one can find in our earlier paper [6]. The diamond film morphology has been studied by scanning electron microscope (SEM), Jeol JSM-820 operating at a voltage of $25 \mathrm{kV}$.

In order to enhance the diamond nucleation density, the silicon substrate had been scratched with a diamond paste before the deposition process was started.

\subsection{Raman spectroscopy}

The Raman spectra were recorded at room temperature in backscattering geometry using Renishaw inVia Raman spectrometer. The $488 \mathrm{~nm}$ argon laser line was used for excitation. The applied power of the laser beam before focusing with $\times 50$ objective was less than $6 \mathrm{~mW}$. An edge filter was used to stray Rayleigh light rejection. The instrumental resolution was better than $2 \mathrm{~cm}^{-1}$. The spectral parameters of Raman bands, such as Raman shift, intensity, integral intensity, and FWHM (full width at half maximum) were determined using fitting procedure.

\subsection{Cathodoluminescence (CL)}

The CL analysis was performed using a FESEM apparatus modified in order to take CL spectra. The CL emission spectra were registered at room temperature using a grating spectrometer equipped with a charge-coupled device camera. The accelerating voltage of the electron beam was $20 \mathrm{kV}$. Two gratings were used, giving a spectral resolution of $0.5 \mathrm{~nm}$ or $0.2 \mathrm{~nm}$ in the case of high resolution spectra. The magnification was varied between $\times 10,000$ and $\times 40,000$ and the acquisition time between $30 \mathrm{~s}$ and $60 \mathrm{~s}$.

\subsection{Thermoluminescence (TL)}

The TL experiments were carried out with help of RISO TL/OSL-DA-20 luminescence reader, equipped with EMI9235QA photomultiplier and beta and alpha radiation sources. Such equipment is commonly used in the field of luminescence dating [7], but it can be also applied for TL measurements in material studies. The sample was put on stainless steel discs (9.7 $\mathrm{mm}$ in diameter) and silicon oil was used for adhesive. The sample was kept and operated in light tight conditions. Prior to the irradiation it was preheated up to $450{ }^{\circ} \mathrm{C}$. The TL was measured without any optical emission filters with a heating rate of $1 \mathrm{~K} / \mathrm{s}$ in argon atmosphere. The nominal dose of beta radiation of 940 Gy applied from ${ }^{90} \mathrm{Sr} /{ }^{90} \mathrm{Y}$ source in standard arrangement was estimated on the basis of the dose rate, as calibrated for quartz grains with the diameter of the order of $200 \mu \mathrm{m}$ [8]. The alpha irradiation was realized using incorporated ${ }^{241} \mathrm{Am}$ foil source of nominal activity of $10.7 \mathrm{MBq}$ under vacuum conditions (the chamber was evacuated below $10 \mathrm{~Pa}$ ). The $27 \mathrm{~Gy}$ of the nominal dose of alpha radiation was calculated assuming $45 \mathrm{mGy} / \mathrm{s}$ of the dose rate, as expected for fine quartz grains with the diameter of $10 \mu \mathrm{m}$.

\section{Results}

Fig. 1 shows SEM micrographs of the diamond films deposited in this study. Both samples were deposited at very similar conditions in order to test the preparation of diamond layers having reproducible thermoluminescent properties. The small distance between the filament and substrate resulted in a microsized diamond films with thicknesses of $2.5 \mu \mathrm{m}$ (Fig. 1a) and $3.5 \mu \mathrm{m}$ (Fig. 1b).

Diamond films synthesized by CVD with methanol and other carbon sources, in general, contain disordered graphitic carbon phase [9]. To check diamond quality and detect other carbon phase in the diamond layer the best tools for this 


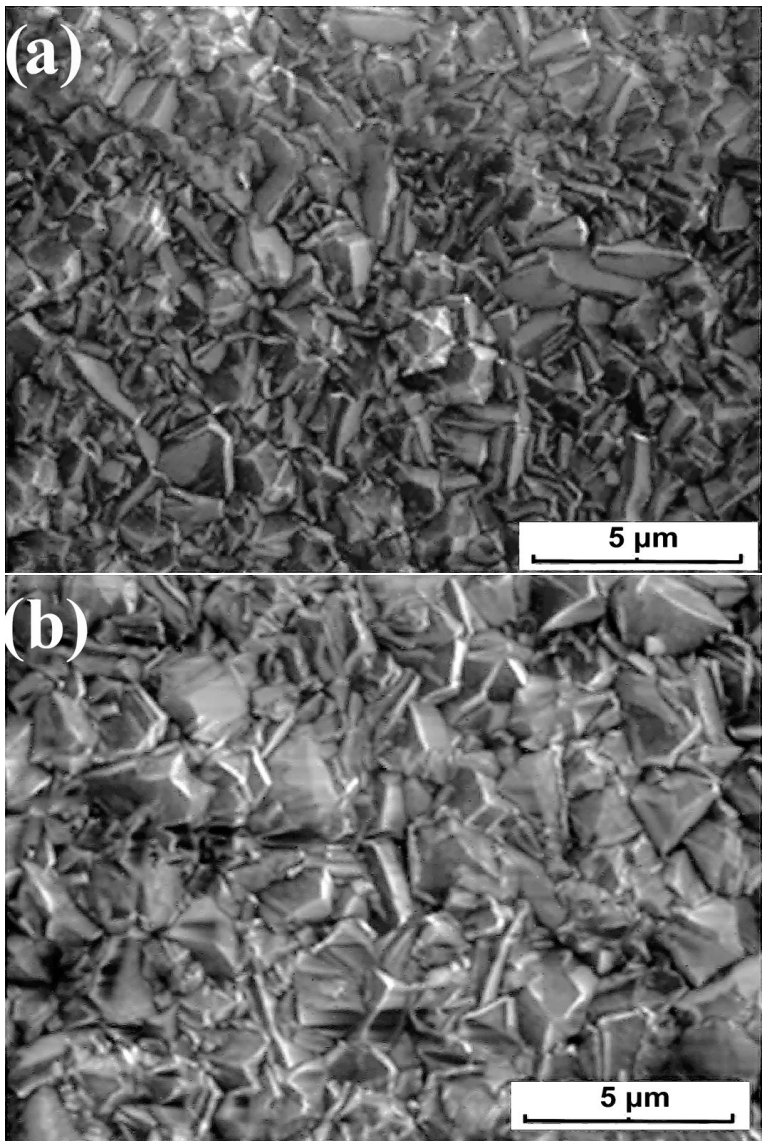

Fig. 1. The morphology of diamond films: (a) $2.5 \mu \mathrm{m}$, (b) $3.5 \mu \mathrm{m}$ thick.

task is Raman spectroscopy because each carbon phase has its own characteristic Raman spectrum. It is known that cross-section of Raman scattering for graphitic phase is much higher than that for diamond phase, thus allowing even a small amount of graphitic carbon to be recognized in diamond films. Fig. 2 presents the Raman spectra of diamond layers studied in this work.

Both Raman spectra show very similar feature, e.g. diamond Raman peaks are centered at $1332.25 \mathrm{~cm}^{-1}$ which is typical of diamond monocrystal. Both diamond samples contain some amounts of $\mathrm{sp}^{2}$-hybridized carbon phase as it is evidenced by weak broad band peaked at $1510 \mathrm{~cm}^{-1}$ (Fig. 2).

Only small differences are observed in full widths at half maximum (FWHM) of diamond Raman lines which are $9.8 \mathrm{~cm}^{-1}$ and $8.9 \mathrm{~cm}^{-1}$ for

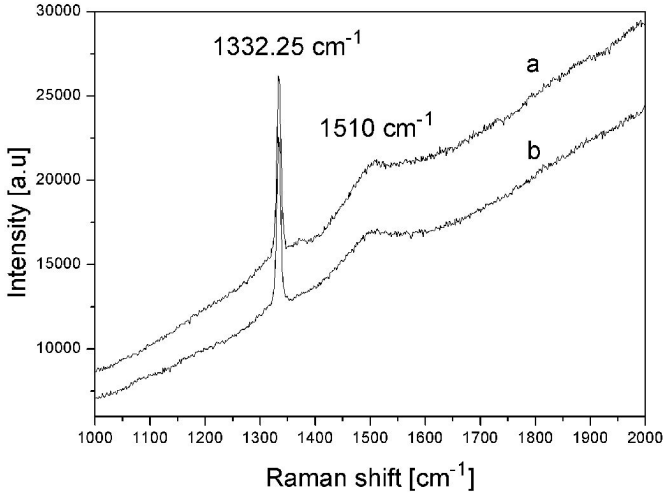

Fig. 2. Raman spectra of the layers presented in Fig. 1.

sample $\mathrm{a}$ and $\mathrm{b}$ respectively. It means that from Raman point of view, the both diamond layers are of similar quality. The statement seems to be confirmed by CL spectra shown in Fig. 3.
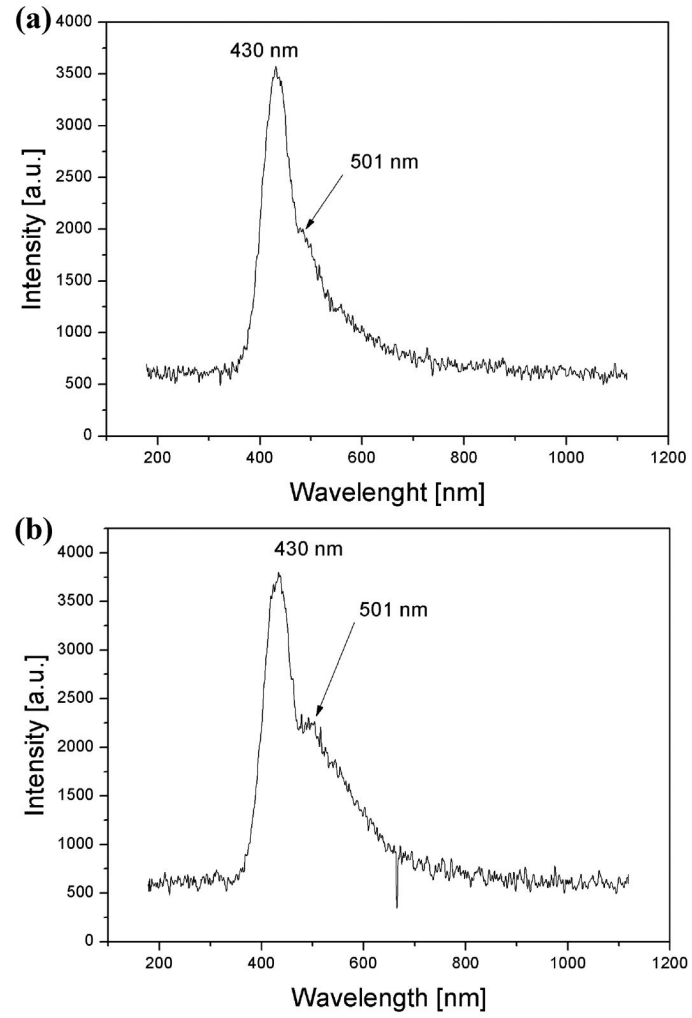

Fig. 3. CL spectra of CVD diamond films presented in Fig. 1.

Both spectra show intense peaks at $430 \mathrm{~nm}$ well-known as band-A and less pronounced peak at $501 \mathrm{~nm}$ known as $\mathrm{H} 3$-center $(\mathrm{N}-\mathrm{V}-\mathrm{N})$, 
originating from dislocations and nitrogen atom inclusions, respectively [10, 11]. Takeuchi et al. [11] reported that the band-A emission from diamond films grown by MWCVD is observed at incoherent grain boundaries and dislocations located inside the nonepitaxial crystallites. Thermoluminescence (TL) is also the method used for defect identification and is usually observed in chemical vapor deposition (CVD) diamond samples that were previously exposed to ionizing radiation. After heating the specimen above the temperature at which the irradiation was carried out, the light emission intensity measured as a function of temperature provided a distinctive TL glow curve. Fig. 4 presents TL glow curves for samples, previously $\beta$-irradiated, presented in Fig. 1.

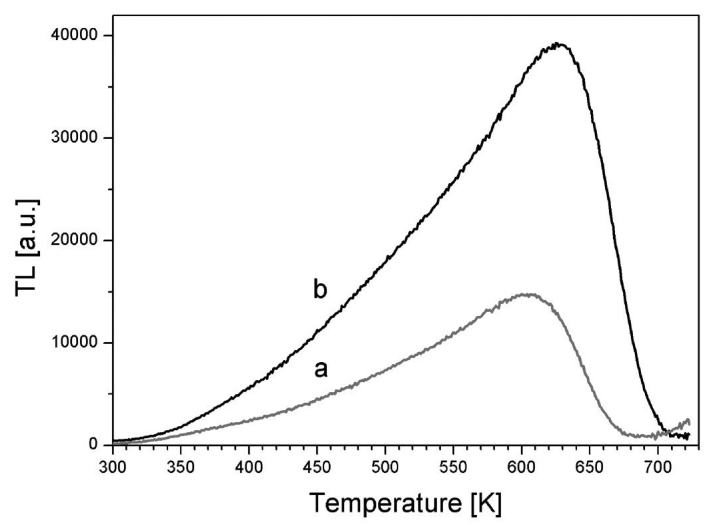

Fig. 4. Glow curves of $\beta$-irradiated samples presented in Fig. 1.

The glow curve gives information regarding the charge trapping and transfer processes resulting in radiative recombination [12]. Localized trapping levels or traps in diamond may be formed inside the wide $(5.5 \mathrm{eV})$ band gap by impurities, doping atoms or lattice defects. On the other hand, the CVD diamond growing conditions may have a strong effect on the morphological properties, crystalline structure, grain boundaries and defect concentration, affecting the number, and distribution of the trap energy levels in CVD diamond films. Therefore, the shape of TL glow curve and peak structure depend strongly on the growth conditions and defects existing in the CVD diamond, making the TL phenomenon a relatively

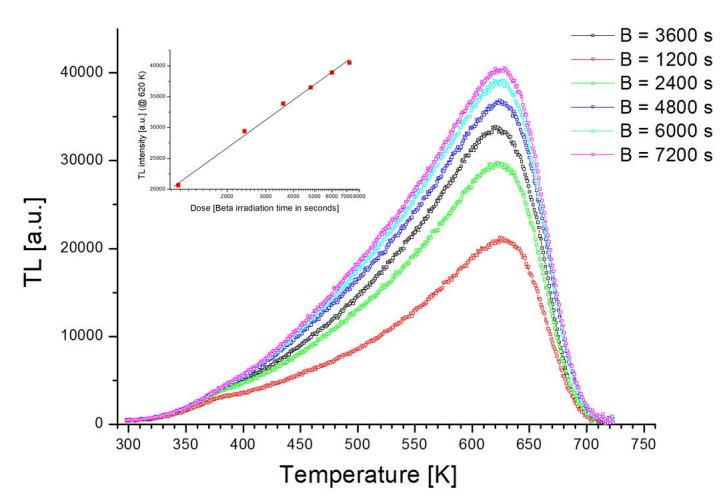

Fig. 5. TL glow curves for the sample from Fig. 1b as a function of $\beta$-dose irradiation. The inset represents the dependence of TL peak intensity vs. irradiation dose.

complex process to understand and replicate for TL dosimetry (TLD) applications. The intention of our experiment was the synthesis of diamond layers having reproducible properties. As it is seen from Fig. 4, both TL curves show similar shape and peak positions which confirms our previous observation from Raman and CL measurements. The differences concern only TL peak intensities which is understandable because the samples have different thicknesses and TL phenomena are volume dependent.

Fig. 5 shows glow curves obtained for sample $b$ for different $\beta$-irradiation doses. The linear increase of the $620 \mathrm{~K}$ peak intensities in the whole dose range clearly evidences the reliable dosimetric properties of this peak, despite the low film thickness.

Similar measurement have been done for the sample from Fig. 1a. The shapes of the glow curves clearly indicate that in TL mechanism, except the $0.83 \mathrm{eV}$ trap, more shallow traps must be also involved. This means that TL mechanism in case of CVD diamond layers is not one-trap onerecombination center process [13].

\section{Discussion}

As it is seen from Fig. 1, both diamond layers are polycrystalline in nature and are composed from small, randomly oriented microcrystals with 
well-defined crystalline features. This observation is in agreement with Raman measurements because in principle, the Raman spectra of both layers (Fig. 2) are very similar and characterized by single sharp peaks at $1332.25 \mathrm{~cm}^{-1}$ that correspond to the $\mathrm{C}-\mathrm{C} \mathrm{sp}{ }^{3}$ bonds. The presence of a broader band around $1510 \mathrm{~cm}^{-1}$ of very low intensity can be attributed to the presence of graphitic carbon impurities ( $\mathrm{sp}^{2}$ carbon). The concentration of nondiamond phase admixture was estimated using the method described in the literature [9] and ranged from $1.9 \%$ to $2.1 \%$ which indicates high-quality diamond films [9] with very small amount of impurities. Raman spectra of the samples in this report show the strong diamond peak and weak graphitic peak. The luminescence background levels of the Raman spectra changed according to the intensities of CL (Fig. 3).

Our diamond layers were not intentionally nitrogen doped but the unintentional incorporation of nitrogen generally occurs in such forms of synthetic diamond as chemically vapor deposited (CVD) diamond thin films due to background elements in the environment of the growth chamber or impurities in the fed gases. In practice, even in high-quality CVD diamond films [14], nitrogenrelated optically active centers have been observed in some cases by means of cathodoluminescence. The identification and characterization of these defects are essential in optimizing the synthesis parameters and the properties of synthetic diamond

\section{Conclusions}

Synthetic diamond grown by CVD is a very attractive material for TLD applications. To achieve the large potentialities, such as soft-tissue equivalence, chemical stability, and non-toxicity, the growth of CVD diamond films with tailored TL properties for radiation dosimetry is necessary. The presence of structural defects and compositional impurities, which depend on the growth conditions, may affect the TL response of the CVD diamond film. We have provided the evidence that the intensity of TL glow curve changes linearly as a function of logarithm of irradiation dose. The shape of TL curves indicates that thermoluminescence process is not one-trap one-recombination center process. It is expected that the HFCVD technique would provide CVD diamond samples with a more reliable TL behavior.

The TL glow curve structure may be ascribed to nitrogen-vacancy complexes caused by traces of nitrogen or other contaminants that modify the film morphology and crystallinity what is in agreement with CL spectroscopy measurements. Further studies are necessary for a conclusive assignment of the TL peaks to particular defects or centers.

\section{References}

[1] KHAn F.M., GiBBons J.P., Khan's the physics of radiation therapy, Lippincott Williams \& Wilkins, 2014.

[2] Lansley S., Betzel G., Baluti F., Reinisch L., MeYer J., Nucl. Instrum. Meth. A, 607 (2009), 659.

[3] Piliero M.A., Hugtenburg R.P., Ryde S.J.S., Oliver K., Radiat. Phys. Chem., 104 (2014), 10.

[4] Brahme A., Comprehensive Biomedical Physics, Elsevier Science, 2014.

[5] Benabdesselam M., IACConi P., Briand D., ButLER J., Diam. Relat. Mater., 9 (2000), 1013.

[6] Fabisiak K., Torz-Piotrowska R., Staryga E., Szybowicz M., PAPROCKI K., BANASZAK A., PoPIElARski P., Mater. Sci. Eng. B-Adv., 177 (2012), 1352.

[7] OczKowski H.L., PrZegietKa K., Radiat. Meas., 29 (1998), 435.

[8] Przegietka K., Chruscinska A., Radiat. Phys. Chem., 104 (2014), 118.

[9] FAbisiak K., SZREiber M., Uniszkiewicz C., Runka T., Kasprowicz D., Cryst. Res. Technol., 45 (2010), 167.

[10] Wang C., Irie M., Ito T., Jpn. J. Appl. Phys., 40 (2001), L212.

[11] Takeuchi D., Watanabe H., Yamanaka S., OKUSHI H., SAWADA H., ICHINOSE H., Sekiguchi T., KaJimura K., Phys. Rev. B, 63 (2001), 245328

[12] Chen R., MCKeEVER S.W., Theory of thermoluminescence and related phenomena, World Scientific, 1997.

[13] Meléndrez R., Chernov V., MaY P., CAStaneda B., Pedroza-Montero M., BarbozaFlores M., Phys. Status Solidi A, 206 (2009), 2103.

[14] Watanabe H., RI S.-G., Yamanaka S., Takeuchi D., OKushi H., New. Diam. Front. $C$. Tec., 12 (2002), 1. 